\title{
UNA (BREVE Y NO MUY SISTEMÁTICA) APROXIMACIÓN A LA NOCIÓN DE AGENCIA DESDE LA VULNERABILIDAD'
}

\author{
A (brief and not very systematic) approach to the notion \\ of agency from vulnerability
}

\author{
María Martínez \\ UNED (España)
}

Palabras clave

Agencia Vulnerabilidad Paradoja

Keywords

Agency

Vulnerability

Paradox

RESUMEN: Este texto presenta el número monográfico «Agencias desde la vulnerabilidad». Frente a la concepción hegemónica de vulnerabilidad que niega la agencia, esta presentación y los artículos de este número invitan a pensar qué sucede cuando confrontamos esos conceptos. El resultado es la necesidad de repensar la noción de agencia y, con ella, los conceptos de acción y de sujeto de la acción. Además del trabajo de Saba Mahmood que se recoge en la sección fundamentales, el número cuenta con las contribuciones de Paola Rebughini, Jane Freedman, Itziar Gandarias, María Santacruz Giralt, Ariana S. Cota, Aurora Álvarez Veinguer y Luca Sebastiani, y Carolina Meloni, a los que se les suma el papel crítico de Álvaro Villar Baile.

ABSTRACT: This text presents the special issue "Agencies from situations of vulnerability". This presentation and the articles of this special issue challenge the hegemonic notion of vulnerability that denies agency and invite to think about what happens when vulnerability and agency are confronted. The result is the need to re-think the notion of agency, and along with it, the concepts of action and subject of action. Besides Saba Mahmood's work that is gathered in the fundamentals section, the issue assembles the contribution from Paola Rebughini, Jane Freedman, Itziar Gandarias, María Santacruz Giralt, Ariana S. Cota, Aurora Álvarez Veinguer and Luca Sebastiani, and Carolina Meloni. The critical paper by Álvaro Villar Baile also joins the issue.

1 Estas breves notas han sido producidas en dos contextos de investigación que merecen ser citados: por un lado, mi investigación postdoctoral financiada por el programa de perfección de personal doctor del Gobierno Vasco (2016-2019), que realicé en la Universidad de California, Santa Barbara (USA); y, por otro lado, el proyecto del plan de I+D+i del Gobierno de España: «Desapariciones. Estudio en perspectiva transnacional de una categoría para gestionar, habitar y analizar la catástrofe social y la pérdida (CSO 2015-66318-P) que alimenta institucionalmente, pero principalmente ha habilitado espacios colectivos de discusión. Entre ellos me gustaría destacar que muchas reflexiones que se presentan en estas páginas han sido fruto de discusiones con mis colegas de este proyecto. Agradezco el intercambio especialmente a Gabriel Gatti, Ignacio Irazuzta, Ander Mendiguren, Ivana Belén Ruiz Estramil y Estela Schindel.

Correspondencia a / Correspondence to: María Martínez. UNED-Departamento de Sociología III. C/ del Obispo Trejo, 2. 28040 Madrid - mariamartinez@poli. uned.es - http://orcid.org/0000-0001-9337-3225.

Cómo citar / How to cite: Martínez, María (2019). "Presentación. Una (breve y no muy sistemática) aproximación a la noción de agencia desde la vulnerabilidad»; Papeles del CEIC, vol. 2019/1, papel 205, 1-9. (http://dx.doi.org/10.1387/pceic.20616)

ISSN 1695-6494 / (c) 2019 UPV/EHU 
Agencia y vulnerabilidad son dos conceptos que han sido profusamente pensados, pero por separado: la literatura sobre la agencia desde diferentes disciplinas y marcos teóricos (sociología, feminismos, estudios postcoloniales...) es inabarcable. También lo son, especialmente en los últimos tiempos, las discusiones sobre el concepto de vulnerabilidad, imposibles de listar y reseñar. No nos detendremos en esa bibliografía pensada por separado, pues lo que interesa en este número monográfico - tanto en lo teórico como en lo empírico-, y lo que intentaré hacer en esta presentación, es prestar atención a qué sucede cuando se cruzan dos conceptos que vienen a proponer prácticamente lo contrario el uno del otro. El primero, la agencia, se ha pensado como un imposible a causa del segundo, la vulnerabilidad. Así es, agencia y vulnerabilidad forman un oxímoron, pues al menos en sus acepciones más hegemónicas, la vulnerabilidad anula (o al menos limita a su mínima expresión) la capacidad de acción.

En ese cruce vulnerabilidad-agencia, el impulso común es explicar la imposibilidad de la segunda cuando la primera es tomada desde su concepción hegemónica. Esto es, y ya lo he dicho, la vulnerabilidad, al ser entendida como pasividad, debilidad, dependencia, como posibilidad de ser herido (Gilson, 2014; Soulet, 2005) supone la imposibilidad o al menos la limitación del ejercicio de la agencia. Aunque este impulso no es erróneo y de hecho explica bien el choque entre estos dos conceptos es, no obstante, objetable, pues carga la explicación del lado de la vulnerabilidad y las soluciones, en su caso, también deben pasar por ella: la vulnerabilidad o es condición negativa de la agencia («los vulnerables» no la tienen) o, en una perspectiva más progresista, es algo celebrable cuando ocurre (Weheliye, 2014). Al igual que los textos que componen este número, en esta presentación me sitúo contra este impulso habitual y me pregunto no por cómo los atributos de la vulnerabilidad hacen que esta chirrie cuando se enfrenta a ella la noción de agencia, sino por los límites teóricos de la concepción de agencia que se revelan cuando la confrontamos con la de vulnerabilidad. La pregunta no es, entonces, por las agencias de "los vulnerables», sino la agencia o las agencias desde la vulnerabilidad, por la agencia «en situación de vulnerabilidad» (Châtel y Soulet, 2003). Esto permite, en última instancia, hacer pensable lo que hasta ahora no lo era: que la vulnerabilidad es la condición de posibilidad de otras formas de agencia y acción (Butler, 2015 y 2017).

El concepto de agencia es un concepto bien moderno, como lo trabaja Paola Rebughini en su artículo. Como concepto moderno que es, la agencia se sostiene en unas concepciones particulares de la acción y del sujeto de la acción, muy normativos. La concepción normativa de la acción es inseparable de una concepción concreta del sujeto: el sujeto moderno, el humano pleno, el ciudadano. Es precisamente la alineación entre esa noción de agencia y de acción con esa concepción del sujeto la que dificulta, incluso imposibilita, no sólo pensar la agencia de los vulnerables, sino principalmente atender a las agencias que se pueden desarrollar desde la vulnerabilidad, que al no ajustarse a nuestras nociones de acción y sujeto de la acción no somos capaces de aprehender. Así, la noción de agencia se apoya en algunos atributos que entremezclan la concepción normativa de la acción y de su sujeto de la acción correlativo; haré el recorrido por los cuatro que considero más significativos y que abren el diálogo de este número monográfico.

El primer atributo de la agencia es que es propiedad del sujeto; la expresión «tal sujeto tiene agencia» es común. La agencia se entiende que es propiedad de un sujeto dado. Es el sujeto quien, dependiendo de las condiciones socio-estructurales en las que se encuentra, tiene o 
no agencia. Existen, por ello, sujetos que pueden tener una agencia (casi) plena, aquellos sujetos liberados o menos sujetados, y otros que, dadas sus condiciones de sujeción y dominación, son despojados de esa propiedad o que tienen unas constricciones tan fuertes que su capacidad de acción es casi inexistente. La tensión agencia-sujeción es precisamente el foco de análisis del trabajo de Santacruz presente en este monográfico. Esta forma de entender la agencia escinde sujeto y acción; presupone sujetos que pre-existen a la acción y que, una vez constituidos, despliegan su acción (García Selgas, 2016: 84). Dos cuestiones emergen en torno a este atributo de la agencia cuando confrontamos esta noción con la de vulnerabilidad. La primera, que "los vulnerables» -emparentados con los subalternos (Spivak, 1988), con víctimas (Gatti, 2016 y 2017) y con otros sujetos "sin» (Rancière, 1995)— no son sujetos, al menos no sujetos à part entière $y$, por tanto, han de carecer de agencia. La segunda, que el problema no se soluciona, sino más bien se enquista, preguntándonos si los vulnerables pueden tener o tienen agencia, pues la respuesta reforzará la misma lógica de un sujeto que posee (o no) agencia y que ese sujeto precede a la acción, no se constituye con, a partir o a través de esa acción (Arnold, 2015; Butler, 2017).

Y es que la agencia, y este es el segundo atributo de esta noción, no es posesión de un sujeto cualquiera, sino del sujeto normado; podríamos decir del ciudadano (Gatti, 2016) que es nuestro referente de sujeto -imbricado, no lo olvidemos, con una figuración concreta del humano pleno (Weheliye, 2014; Wynter, 2003)-, que se caracteriza por ser racional, autónomo, estratégico. Quien quede fuera de lo que hace a ese sujeto normado, ya lo he indicado, carecerá de acción. O mejor dicho, la expulsión de esos sujetos no-normativos se hará precisamente a través de la negación de su capacidad de hacer. Esto revela cómo esta forma de pensar sujeto y acción de manera escindida quiebra, pues una es condición de posibilidad de la otra y la otra de la una. La noción de vulnerabilidad, incluso la de «sujetos» vulnerables, nos interroga sobre qué sucede cuando no hay sujeto normado; basta pensar para tomar consciencia de ello en el largo listado de vulnerables sobre los que trabajan los textos de este número: mujeres refugiadas (Freeman), mujeres subsaharianas en tránsito (Gandarias), pandilleras (Santacruz), activistas expuestas a la violencia institucional (Cota), personas desahuciadas (Álvarez y Sebastiani), jóvenes sin empleo o con empleos precarios (Rebughini). Pero nos interroga aún más cuando ni siquiera hay sujeto (es el caso del texto de Meloni sobre desaparecidos en Tucumán, Argentina). ¿Cómo pensar entonces la agencia si el sujeto que ha de poseerla no es considerado sujeto e incluso cuando carece de su marca mínima de sujeto: el cuerpo? No es posible; no bajo esta noción de agencia.

El tercer atributo de la noción de agencia es que para esta hay un solo tipo de acción posible. La agencia para ser pensada como tal tiene que tomar una forma de acción que no es otra que la del modelo weberiano de acción racional con arreglo a fines (Das, 2008; Dubet, 2010). Es acción racional, intencional, voluntaria, estratégica, la que pone en marcha el sujeto moderno, el humano pleno, el ciudadano. Es acción propia de nuestros regímenes de visibilidad, reconocible para nuestros marcos, por seguir la expresión de Butler (2009). Lo que queda fuera de eso no es acción. Vemos, de nuevo, cómo la escisión sujeto-acción quiebra: no es que el sujeto normativo despliegue sólo este tipo de acción, sino que todo su actuar será entendido como acción. Consecuentemente, las acciones de los vulnerables no serán ni reconocidas (Bierria, 2014), ni reconocibles aun cuando su acción sea la racional con arreglo a fines; serán, por tanto, desagenciados. Lo dice mucho mejor Veena Das: «Todas las otras formas del ser social -la mujer occidental, tanto como la gente no occidental- son comprendidas en cuanto a una carencia, una desviación con respecto a la acción típico ideal repre- 
sentada por el paradigma de la acción racional» (2008: 197). Así, la vulnerabilidad plantea a la agencia y la acción un dilema similar al que sugería Spivak (1988) en torno al habla del subalterno ${ }^{2}$. Si el vulnerable actúa según el modelo normativo de acción del sujeto pleno, si es que esto es tan siquiera posible, dejará de ser vulnerable ${ }^{3}$; si actúa según otros modos de acción, esta no será reconocida (Beirria, 2014) pues queda fuera de nuestro "registro de lo sensible» (Rancière, 2009). Y este quedar fuera de nuestro "registro de lo sensible» se traduce particularmente en la imposibilidad de analizar desde las ciencias sociales formas de acción que no se ajusten al tipo de acción estandarizado. Varios de los artículos que componen este número buscan hacer posible lo imposible, bien analizando los usos de la vulnerabilidad, incluso reapropiaciones de acciones propias del sistema de dominación, como formas de acción (Freeman, Gandarias), bien atendiendo a formas de acción otras (Cota, Álvarez y Sebastiani, Meloni).

Si algo hace a esa noción de acción particular sobre la que se sostiene el concepto de agencia es que la acción es entendida siempre en términos de transformación, incluso resistencia (Ahearn, 2001; García Selgas, 2016). El texto de Saba Mahmood que reproducimos en la sección "Fundamentales» es clave en esta crítica a la idea de que la acción ha de ser siempre transformadora. La autora desarrolla una crítica a algunas teorías feministas, especialmente los aportes de Butler en torno a la agencia. Para Mahmood el enfoque de Butler, entre otras, sólo permite considerar la agencia y la acción cuando transforman. Esto obvia formas de acción que reproducen 0 , al menos, permiten habitar las normas que nos constituyen (Mahmood, 2005), aunque sean estas dominadoras. La acción que reproduce normas no es acción, pues la acción para serlo ha de transformar. Contra esta idea trabajan particularmente los textos de Gandarias y Freedman.

El sujeto normativo de la agencia es un sujeto que hace el mundo y que haciéndolo, se hace a sí mismo (Das, 2008). Esta acción transformadora puede ser, por supuesto, individual, pero la acción colectiva es la que mejor encaja con esta idea de que la acción para ser tal ha de transformar, incluso resistir ante un poder o un sistema de dominación. Y ello porque la acción colectiva y política supone un sujeto que rehace el mundo modificando las normas que constituyen el mundo y le constituyen a sí mismo. Así sucede en la literatura de movimientos sociales y acción colectiva, sólo capaz de abordar movimientos que buscan transformar o resistir ante el sistema; incapaz de atender a aquellos que desarrollan acciones que, bien les permitan vivir más hábilmente, y quizás con mayor dignidad, en el sistema opresivo, bien les habilitan a generar espacio-tiempos aparentemente fuera del sistema de dominación o, aún, resignificar las identidades que les han sido impuestas. Y esto, que es común en movimientos de víctimas y de "vulnerables» (Lefranc y Mathieu, 2009; Mathieu, 2001; Piven y Cloward, 1979), lo ponen de relieve en este número los trabajos de Álvarez y Sebastiani, y de Cota. Ambos plantean que la acción colectiva desde la vulnerabilidad no siempre responde a las acciones más visibles de los movimientos - acciones públicas que tienen al Estado o al gobierno como objeto-, sino que es necesario estar atentos a formas de acción más cotidianas (Jokinen, 2016), también a acciones que no buscan más que construir un proyecto propio, como analiza Cota. Estas acciones muestran, además, que la vulnerabilidad no es algo ni a

Para una revisión más profunda de esta cuestión, ver Gatti y Martínez, 2017.

Cierta literatura francesa sobre la acción (colectiva) de las víctimas y los vulnerables parece sugerir que ese ha de ser el camino y que, de hecho, debemos tratar a estos sujetos vulnerables como a un actor cualquiera (Lefranc y Mathieu, 2009). 
superar ni que invalide la acción, sino al contrario la condición de posibilidad de otras formas de agencia y acción colectivas.

Entendida en su versión hegemónica y moderna, la agencia es un concepto del que deberíamos deshacernos cuando lo confrontamos con el de vulnerabilidad. No es lo que hacen los textos que reúne este número monográfico. Al contrario, se proponen repensar la noción de agencia no para reivindicarla para "poblaciones vulnerables», sino proponiendo qué agencias -individuales y colectivas, privadas y políticas, reproductoras y transformadoras- son posibles desde la vulnerabilidad.

\section{AGENCIAS INDIVIDUALES DESDE LA VULNERABILIDAD}

Paola Rebughini firma el primer artículo de este número, «A vulnerable generation? Youth agency facing work precariousness». En él analiza cómo vulnerabilidad y agencia no son conceptos opuestos, sino necesariamente entrelazados. Tras un recorrido preciso y sintético por las concepciones teóricas sobre la agencia, se apoya en un análisis de cómo personas jóvenes del área de Milán (Italia) se enfrentan a la falta de empleo y a las condiciones crecientes de precariedad del mercado de trabajo para mostrar que, en situaciones de vulnerabilidad, la agencia deviene una necesidad, una tarea, una actividad imparable. Desde una perspectiva crítica, Rebughini nos alerta de que esa necesidad de reforzamiento de la agencia - que es además individual, nunca resultado de un proceso colectivo- puede terminar reforzando al sujeto moderno, soberano y, por tanto, agente. Así, la vulnerabilidad no es falta de agencia, sino que se convierte en un mandato para performar una agencia que les es negada, un ideal al que aspirar. Una agencia que en su concepción como propia del sujeto moderno y soberano es, además, masculina, con las imposibilidades que esto supone particularmente para las mujeres jóvenes en situaciones de falta de empleo y de precariedad laboral.

Los usos de la vulnerabilidad constituyen el objeto del artículo de Jane Freeman, "The uses and abuses of "vulnerability" in EU asylum and refugee protection: protecting women or reducing autonomy?». La autora argumenta que el concepto de vulnerabilidad hegemónico - como dependencia, debilidad, posibilidad de ser herido y de sufrir violencias - impregna las políticas europeas de asilo y refugio definiendo las percepciones sobre sus solicitantes, especialmente mujeres dado el carácter generizado del concepto. Ser definida como vulnerable da acceso a las protecciones que esas políticas otorgan, pero hacerlo también supone modificar la percepción sobre sus identidades e intereses. Así, el artículo explora los usos, más o menos explícitos, de la categorización como vulnerables por parte de mujeres demandantes de asilo que en ocasiones se pliegan a los mandatos de la «buena» refugiada - vulnerable $y$, por tanto, necesitada de asistencia, pasiva, débil, sin agencia- y en otros subvierten los mandatos que se les imponen. En cualquiera de los dos casos - pliegue o subversión de los mandatos de vulnerabilidad - lo que estas situaciones muestran es la maleabilidad del concepto de agencia, que no es nunca totalmente transformadora.

Es precisamente la resistencia desde la vulnerabilidad el eje de reflexión del artículo de Itziar Gandarias, «Resistir desde la vulnerabilidad: Narrativas de mujeres subsaharianas sobre su tránsito hacia Europa». Haciendo uso de la metodología de producciones narrativas, la autora trabaja con y sobre la narración de tres mujeres subsaharianas en torno a su tránsito ha- 
cia Europa. Su texto se centra en actos de resistencia, en ocasiones menores, siempre en relación con la vulnerabilidad a la que se ven expuestas durante el tránsito. Ese análisis de los pequeños actos de resistencia incide en una idea que inspira este número monográfico: que vulnerabilidad es condición de posibilidad y puede incluso habilitar (otras formas de) agencia. En línea con el trabajo de Mahmood, la autora no entiende la agencia como oposición a los sistemas de dominación sino como el despliegue de acciones - por minúsculas que seanque permiten performar y habitar las normas sociales de manera heterogénea. Así lo hacen las tres mujeres que analiza Itziar Gandarias: usan el sistema de dominación, actúan según las normas que en principio las dominan, como depender de un varón durante el viaje o portar el velo para conseguir su objetivo de llegar a Europa. Así, resistencia como forma máxima de agencia no se opone a vulnerabilidad, sino que la vulnerabilidad (o sus usos, en el sentido de la propuesta de Freedman) permiten formas de resistencia que no transforman el sistema, pero permiten hacer la vida de estos sujetos un poco más vivible.

Las tensiones entre sujeción y agencia en contextos de vulnerabilidad son la materia de reflexión del texto que nos propone María Santacruz Giralt, titulado «Mujeres en pandillas salvadoreñas y las paradojas de una agencia precaria». La autora problematiza una cuestión fundamental en torno a la noción de agencia y es que en organizaciones violentas como las maras o pandillas, la agencia no es un simple ejercicio individual de un sujeto soberano, sino que muestra el resultado de un proceso de sujeción. Si esto es asi en el caso de los varones, el artículo nos invita a preguntarnos si las mujeres pueden desarrollar agencia desde la sujeción cuando en su caso sujeción no las habilita como sujetos, sino como objetos. Las mujeres pandilleras han de apropiarse a la vez que desviarse, explica Santacruz, del «marco productor de un 'pandillero-prototipo'». Acudiendo a la teoría de la performatividad de Butler, la autora sitúa la capacidad de agencia de esas mujeres en su necesaria, aunque imposible de conseguir, repetición constante de ese modelo de pandillero dado que esa inevitable repetición de una identidad que las sujeta les permite marcar distancias y estrategias distintas de las permitidas por el modelo masculino. Esa agencia no es, no obstante, la misma que la hegemónica, la de los varones, sino una agencia siempre precaria.

\section{LA VULNERABILIDAD COMO CONDICIÓN DE POSIBILIDAD DE (OTRAS) AGENCIAS COLECTIVAS}

Los dos textos siguiente trabajan ya no sobre agencias en contextos grupales (como el de pandillas o maras sobre los que trabajaba el último texto reseñado), sino sobre agencias colectivas. De hecho, en ambos el análisis versa sobre movimientos o formas de acción colectiva muy cercanas, y no sólo geográficamente: el primero sobre Stop Represión Granada, el segundo en torno a las luchas contra los desahucios en esa misma ciudad.

La propuesta de Ariana S. Cota, "Procesos de agenciamiento junto a Stop Represión Granada y un ejercicio de autoetnografía vulnerable», es doble en su tratamiento de la agencia y la vulnerabilidad. Por un lado, analiza los procesos de agenciamiento del colectivo Stop Represión Granada, colectivo al que ella misma pertenece, no sólo a través del análisis de acciones visibles y de oposición al poder, sino particularmente de acciones hacia el mismo grupo para generar un proyecto propio haciéndose eco de los trabajos de la antropóloga Sherry 
Ortner. Por otro lado, la autora hace un planteamiento de su condición vulnerable -en tanto expuesta- por su doble afiliación académica (como doctoranda que hace una tesis) y como activista del movimiento sobre el que trabaja. En lugar de reafirmar la capacidad de acción de los vulnerables, lo que la autora propone es, además de una noción de agencia que remite a lo colectivo y no a lo individual, desafiar la idea de que para desarrollar agencia se han de superar las vulnerabilidades. Al contrario, sólo asumiendo la condición vulnerable se puede desarrollar agencia y colectivo mediante el «estar juntas» y el «hacer cosas juntas».

En una línea similar se mueve el texto de Aurora Álvarez Veinguer y Luca Sebastiani, "Una década de luchas contra los desahucios. De la vergüenza y la soledad a los agenciamientos cotidianos». Los autores buscan rellenar un vacío en los trabajos sobre desahucios, demasiado centrados en incidir en la vulnerabilidad de las personas desahuciadas. Y es que, ya lo decíamos, las teorías de movimientos sociales y acción colectiva tienen dificultades para pensar la acción colectiva de "los vulnerables». A través de una etnografía colaborativa entre los dos investigadores y el grupo con el que trabajan, resignifican la idea de acción colectiva al analizar no sólo las acciones más visibles (ej. las demandas de las organizaciones de personas desahuciadas ante las autoridades gubernamentales o económicas contra los procesos de desahucio), sino también las acciones más cotidianas, entendidas como forma de hacer frente a la vulnerabilidad estructural y que permiten activar un proceso de subjetivación política desde el sufrimiento y la vulnerabilidad. Estas reflexiones conectan de nuevo con la idea de que la vulnerabilidad no es lo opuesto a la agencia, sino incluso su condición de posibilidad.

\section{AGENCIAS FANTASMALES}

El texto que cierra el número monográfico, «Cadáveres insumisos: hacia una ética del asedio y de la hospitalidad", de Carolina Meloni, trabaja desde otra disciplina y desde temas aparentemente distantes (los desaparecidos en Tucumán, Argentina), en torno a agencias imposibles ¿Es posible la agencia sin sujeto cuando se carece incluso de la base constitutiva de este -el cuerpo-? Con base en un análisis de los trabajos de recuperación en el Pozo de Vargas (Tucumán, Argentina) de restos de personas desaparecidas en la última dictadura militar, la autora nos empuja a pensar en la agencia de los restos, de cuerpos altamente vulnerabilizados. Meloni se pregunta por lo que esos restos hacen en otros, aunque ese hacer se limite a hacer duelo. Pero la acción de esos restos no se reduce a lo que producen o hacen hacer a otros $^{4}$; la aparición misma del cuerpo muerto hace que este recobre la agencia política que le fue arrebatada a través de la práctica de la desaparición forzada. No disponen, por supuesto, de la agencia política previa a la desaparición, pero la simple aparición de esos cuerpos-restos, de esos espectros o revenants, asedia a la comunidad política, que se ve obligada a repensarse $y$, en algunos casos, a desarrollar un efecto de hospitalidad.

El monográfico cuenta, además, con la reseña del documental «Vivir y otras ficciones» que firma Álvaro Villar Baile. El análisis de ese documental muestra las agencias desde la vulnerabilidad ontológica — diversidad funcional, soledad y enfermedad psiquiátrica - en diferentes

4 En este sentido se puede consultar el trabajo de Irazuzta (2017) que plantea que la búsqueda de desaparecidos en México activa un hacer por parte de quiénes los buscan. 
declinaciones: en un caso como habitabilidad de la vulnerabilidad constituyente de manera un tanto resignada, en el otro desde la politización de la propia vulnerabilidad como forma de agencia.

\section{BIBLIOGRAFÍA}

Ahearn, L.M. (2001). Language and Agency. Annual Review of Anthropology, 30, 109-137.

Arnold, L. (2015). The reconceptualization of agency through ambiguity and contradiction: Salvadoran women narrating unauthorized migration. Women's Studies International Forum, 52, 10-19.

Bierria, A. (2014). Missing in Action: Violence, Power, and Discerning Agency. Hypatia, 29(1), 129-145.

Butler, J. (2009). Frames of war. Londres-Nueva York: Verso.

Butler, J. (2015). Notes toward a performative theory of assembly. Cambridge: Harvard University Press.

Butler, J., Gambetti, Z., Sabsay, L. (2017). Vulnerability in resistance. Durham: Duke University Press.

Châtel, V., y Soulet, M-H. (Eds.) (2003). Agir en situation de vulnérabilité. Laval: PUL.

Das, V. et al. (2008). Sujetos de dolor, agentes de dignidad. Bogotá: Pontificia Universidad Javeriana.

Dubet, F. (2010). Sociología de la experiencia. Madrid: CIS y Universidad Complutense de Madrid.

García Selgas, F. (2016). Pensar la agencia en crisis: de la acción y la agencia a la actancia. En B. Tejerina y G. Gatti (Eds.), Pensar la agencia en la crisis (pp. 83-102). Madrid: CIS.

Gatti, G. (2016). La hipótesis V. Las víctimas y su victoria sobre el poderoso (y muy agencial) ciudadano. En B. Tejerina y G. Gatti (Eds.), Pensar la agencia en la crisis (pp. 145-164). Madrid: CIS.

Gatti, G. (2017). Un mundo de víctimas. Barcelona: Anthropos-Siglo XXI.

Gatti, G., y Martínez, M. (2017). El ciudadano-víctima. Notas para iniciar un debate. Revista de Estudios Sociales, 59, 8-14.

Gilson, Erinn (2014). The ethics of vulnerability: a feminist analysis of social life and practice. Nueva York: Routledge.

Irazuzta, I. (2017). Aparecer desaparecidos en el norte de México: las identidades de la búsqueda. En G. Gatti (Ed.), Desapariciones. Usos locales, circulaciones globales (pp. 141162). Bogotá: Siglo del Hombre Editores y Universidad de los Andes.

Jokinen, E. (2016). Precarious everyday agency. European Journal of Cultural Studies, 19(1), 85-99. 
Lefranc, S., y Mathieu, L. (2009). Mobilisations de victimes. Rennes: PUR.

Mahmood, S. (2005). Politics of piety. The Islamic revival and the feminist subject. Princeton: Princeton University Press.

Mathieu, L. (2001). Mobilisations de prostituées. París: Belin.

Piven, F., y Cloward, R. (1979). Poor People's Movements: Why They Succeed, How They Fail. Nueva York: Vintage.

Rancière, J. (1995). La mésentente. París: Galilée.

Rancière, J. (2009). El reparto de lo sensible. Estética y política. Santiago de Chile: LOM.

Soulet, M-H. (2005). La vulnérabilité comme catégorie de l'action publique. Pensée plurielle, 2(10), 49-59.

Spivak, G. (1988). Can the Subaltern Speak? En C. Nelson y L. Grossberg (Eds.), Marxism and the Interpretation of Culture (pp. 271-313). Basingstoke: Macmillan Educat.

Weheliye, A.G. (2014). Habeas Viscus: Racializing Assemblages, Biopolitics, and Black Feminist Theories of the Human. Durham: Duke University Press.

Wynter, S. (2003). Unsettling the Coloniality of Being/Power/Truth/Freedom: Towards the Human, After Man, Its Overrepresentation-An Argument. The New Centennial Review, $3(3), 257-337$. 